\title{
¿Con sentido o sin sentido? \\ En torno a un argumento de Carnap
}

\author{
Andrei Moldovan \\ Departamento de Filosofía, Lógica y Estética \\ Universidad de Salamanca \\ mandreius@usal.es
}

\section{Introducción}

Entre las concepciones de lo real y los estilos de vida se pueden establecer muchas relaciones. Pero si se quiere enfocar el tema desde la perspectiva de la filosofía analítica, un grupo de autores de la primera parte del siglo $x x$ ofrecen un excelente punto de partida. Consideremos, por ejemplo, el siguiente fragmento del libro de Rudolf Carnap, Philosophy and Logical Syntax (1935), capítulo I:

[...] el sistema de metafísica del Monismo puede ser una expresión de un modo uniforme y armonioso de vida, un sistema Dualista puede ser una expresión del estado emocional de una persona que toma la vida como una lucha eterna; [...] el Realismo es a menudo un síntoma del tipo de constitución denominada por los psicólogos extrovertido [...] el Idealismo, de una constitución opuesta, la denominada tipo introvertido $[\ldots]^{1}$.

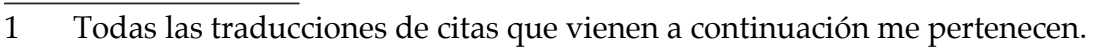


Estas curiosas observaciones de Carnap tienen detrás una visión de la metafísica tanto extraña como radical: "las proposiciones metafísicas no son ni verdaderas ni falsas, porque no afirman nada, no contienen ni conocimiento ni errores" (Carnap, 1935: cap. I). Las aseveraciones de la metafísica son sinsentidos. Pero, aunque no tengan contenido semántico alguno o, en palabras de Carnap, "función representacional", tienen, como todo uso del lenguaje, "una función expresiva". Dicen algo acerca de la psicología de la persona que profiere tales enunciados. En el caso particular de la metafísica, dada su naturaleza, expresan la actitud del metafísico hacia la vida o la realidad.

Carnap comenta en el mismo texto que no espera que su tesis acerca de la naturaleza del discurso metafísico encuentre fácilmente el consenso entre los filósofos, pues sin duda parece legítimo hacerse preguntas como: ¿existen los números? (a diferencia de: ‘qué números existen?'), o ¿existe la rojez? (y no '¿qué cosas rojas existen?'). Una pregunta de esta índole plantea Sócrates en la República de Platón cuando busca la naturaleza de la justicia, y no admite como respuestas meros ejemplos de casos particulares de acciones justas. Este tipo de empeños intelectuales parecen tener propósito y sentido; pero también es legítimo preguntarse, siguiendo a Carnap, si eso es realmente así.

Este tipo de preocupaciones ha recibido mucha atención últimamente, después de un largo periodo de olvido, en el ámbito de la meta-metafísica. La palabra 'meta' en el nombre de esta disciplina indica que se trata de temas metodológicos relativos a la metafísica. Hablar de estos temas es legítimo y esperado teniendo en cuenta la actitud concienciada de los filósofos respecto a la metodología de su disciplina; sin embargo, preocuparse demasiado por la finalidad y la na- 
turaleza de la actividad que se emprende no es un signo de confianza. Como señala Elizabeth Barnes (2009):

Teniendo en cuenta que la metafísica sólo regresó a la corriente filosófica en las últimas décadas del siglo xx (después de largos años en el marco post-positivista), parece haberse 'vuelto meta' mucho más pronto que otras disciplinas. Además, parece haber 'ido meta' en gran medida con el fin de defenderse a sí misma como una disciplina que vale la pena.

Si la disciplina vale la pena o no, depende, en parte, del éxito o fracaso del argumento de Carnap que analizaré en este trabajo.

El debate metaontológico acerca de la naturaleza de las cuestiones ontológicas ha sido y sigue siendo muy influenciado por la filosofía del lenguaje. Históricamente, la teoría verificacionista del significado lingüístico, defendida por los empiristas lógicos, fue la razón principal por la cual se consideró que la metafísica es un discurso sin sentido. Éste es el caso del fragmento de Carnap (1935) citado más arriba. Carnap, Moritz Schilck y otros pensaron en esa época que la teoría verificacionista proporcionaba una base prometedora para descartar las aseveraciones metafísicas como sin sentido². Más

2 El locus clasicus de este argumento es el texto de Carnap, "La eliminación de la metafísica por medio del análisis lógico del lenguaje" (1932), además del capítulo de Carnap (1935) citado anteriormente. En Carnap (1935), el autor aclara que "no incluyo en la metafísica esas teorías -a veces llamadas metafísicas- que tienen por objeto organizar las proposiciones más generales de las distintas regiones del conocimiento científico en un sistema bien ordenado; tales teorías pertenecen en realidad al campo de la ciencia empírica, no de la filosofía". En el comentario final al artículo de 1932, añadido en 1957, Carnap califica aún más su ataque a la metafísica: "Este término ['la metafísica'] se utiliza en este trabajo[...] para el campo del supuesto conocimiento de la esencia de las cosas, que trasciende el ámbito de la ciencia inductiva, fundada empíricamente. Metafísica, en este sentido, incluye sistemas como los de Fichte, Schelling, Hegel, Bergson, Heidegger". 
tarde, cuando la teoría verificacionista perdió su atractivo ${ }^{3}$, fueron los argumentos que Carnap proporcionó en "Empirismo, Semántica y Ontología" (1950) los que se convirtieron en la nueva referencia obligatoria para aquellos que buscaban justificar su escepticismo acerca de la metafísica. Este artículo se discute generalmente como parte de un debate más amplio entre Carnap y Quine en la década de 1950. Es comprensible, por lo tanto, que este debate recibiera mucha atención durante el desarrollo de la meta-metafísica en los últimos años. En la sección 1 describo brevemente los puntos de referencia principales de este debate. En la sección 2 doy algunas razones (inspiradas en la literatura ya existente sobre este asunto) para rechazar el argumento principal que ofrece Carnap en su artículo de 1950 mencionado. Finalmente, en la sección 3 analizo el argumento de Carnap desde una perspectiva meta-semántica actual, en particular desde un punto de vista externista.

\section{Breve descripción del debate Carnap-Quine}

Quine, en “On What There Is" (1948), propone la tesis según la cual el compromiso ontológico de una teoría no se refleja ni en los nombres propios de la teoría (pues los nombres propios, considera Quine, son descripciones definidas encubiertas; y tales expresiones son eliminables mediante la teoría de Russell de las descripciones), ni en los términos generales usados (por razones que tienen que ver con una teoría conductista del significado, que Quine defiende). En cambio, "una teoría se compromete con la existencia de ésas y sólo esas entidades a las que las variables ligadas de la teoría deben ser capaces de referir a fin de que las afirmaciones hechas en la

3 Como resultado de las críticas de, por ejemplo, Carl Hempel (1950). 
teoría sean verdaderas" (Quine, 1948: 13-14). Según Quine, se trata de un criterio para decidir cuáles son los compromisos ontológicos de una teoría. Este criterio no pretende decidir cuáles afirmaciones metafísicas son correctas y cuáles no, y no pretende descartar ninguna posición metafísica como carente de sentido: "la pregunta acerca de qué ontología habría que adoptar sigue en pie, y el consejo obvio es la tolerancia y un espíritu experimental" (1948: 19), escribe Quine. De hecho, Quine toma en serio las posiciones tradicionales en la metafísica. En “On Universals" (1947) y en "Logic and the Reification of Universals" (1953b), Quine sostiene ciertas tesis metafísicas. En breve, según Quine el pensamiento matemático nos compromete con la existencia de las clases, que son universales ${ }^{4}$. Dado que las matemáticas no pueden prescindir de las clases, tampoco pueden prescindir de los universales; sin embargo, dado que quiere evitar comprometerse con una posición platonista acerca de los universales, propone una posición que denomina "conceptualismo". El conceptualismo admite las clases como universales, pero difiere del platonismo en que admite solamente aquellas clases que se introducen por condiciones de pertenencia expresables en el lenguaje (1953b: 126-7).

Carnap, que por lo demás comparte con Quine un punto de vista empirista, da el siguiente paso. Consistente con su actitud antimetafísica", en "Empirismo, Semántica y Onto-

$4 \quad$ Señala Armstrong (1989: 10) que Quine habla de clases como universales, pero este modo de hablar es potencialmente engañoso, pues los universales son repetibles, es decir, tienen instancias, mientras que las clases no lo son. Probablemente debemos interpretar a Quine como sosteniendo que las clases son objetos abstractos, no universales.

5 Carnap se ve obligado a abandonar muchas de las tesis que defendía en los años del Círculo de Viena, pero no abandona su actitud antimetafísica. Wilfrid Sellars recuerda en sus "Reflexiones Autobiográficas" un encuentro con Carnap a finales de la década de 1940: “En ese momento, yo estaba hablan- 
logía" (1950), Carnap presenta sus objeciones a las tesis metafísicas de Quine y en general a la manera en la que éste trata con toda seriedad viejos problemas metafísicos. En particular, Carnap rechaza ciertas consecuencias de carácter metafísico que extrae Quine de su criterio de compromiso ontológico. Según Quine (1948), cuantificar sobre variables que toman como valores números implica un compromiso ontológico. Si cuantificamos sobre números, entonces nos comprometemos con que los números, que son clases, existen (es decir existen realmente, según Quine, en el único sentido en el que se puede decir de algo que existe).

Una premisa fundamental en el argumento de Carnap (1950) es que enunciados como "Los números existen", pueden entenderse de dos maneras. Dice Carnap (1950: 206):

Si alguien desea hablar en su lenguaje sobre un nuevo tipo de entidades, tiene que introducir un sistema de nuevas formas lingüísticas, regidas por nuevas reglas; vamos a llamar a este procedimiento la construcción de un marco [framework] lingüístico para las nuevas entidades en cuestión. $Y$ ahora tenemos que distinguir dos tipos de preguntas de existencia: primero, preguntas acerca de la existencia de ciertas entidades de la nueva clase en el marco introducido; las llamamos preguntas internas; y, segundo, las preguntas relativas a la existencia o la realidad del sistema de las entidades como un todo, llamadas preguntas externas.

do de su Logische Aufbau en mi seminario de Análisis Filosófico, y apenas se había instalado en el coche durante el viaje de regreso cuando empecé a bombardear mi audiencia cautiva con preguntas. Hace tiempo que se me olvidaron los detalles de lo que quería saber, pero recuerdo claramente que su primera reacción fue la de protestar: '¡Pero ese libro fue escrito por mi abuelo!'”. 
Carnap añade que preguntas como " ¿Hay un trozo de papel blanco sobre mi escritorio?" o “¿Acaso el rey Arturo existió de verdad?" tienen respuestas sintéticas y a posteriori, que se pueden averiguar si nos sometemos a cierta investigación empírica. Pero para eso, dice Carnap, tenemos que aceptar previamente "el lenguaje de las cosas", es decir, un marco lingüístico en el que podemos hablar de cosas y eventos espacio-temporales. Las preguntas anteriores son internas a este marco. Consideremos ahora las preguntas como: "¿hay cosas espacio-temporales?”, o “¿existen los números?”. Estas preguntas pueden considerarse de dos maneras: como preguntas internas al "lenguaje de las cosas", o el marco lingüístico de los números (el lenguaje de las matemáticas), respectivamente. Como tal, son preguntas que tienen una respuesta afirmativa trivial. En el caso de la pregunta “¿existen los números?", la respuesta se deduce de enunciados matemáticos verdaderos como: "hay un número superior a 5 e inferior a 7." De hecho, según Carnap "los números existen”, en sentido interno es un enunciando analítico. Carnap considera que su verdad se infiere lógicamente de las reglas semánticas del lenguaje de las matemáticas. Dejando de lado la cuestión de si el enunciado es en realidad analítico o no, sin duda es una verdad trivial. Con lo cual, es un enunciado con sentido.

Pero, sostiene Carnap, estas preguntas y enunciados también se pueden entender como externas a los respectivos marcos lingüísticos. Las aseveraciones de la metafísica tradicional son, según Carnap, externas al marco lingüístico considerado. Por ejemplo, la preferencia de un enunciado como "Los números existen", en el sentido que le quiere dar el metafísico, es externo al marco lingüístico de las matemáticas. El metafísico no se interesa por la existencia de los números en las matemáticas, es decir, no quiere saber si el matemático acepta o postula 
números. El metafísico quiere saber algo diferente: si realmente los números existen. $Y$ en este sentido parte de una pregunta que es externa a las matemáticas.

La primera premisa del argumento de Carnap es que las aseveraciones metafísicas, p. ej. aseveraciones acerca de la existencia de los números, son externas (a las matemáticas, en este caso). La segunda es que toda aseveración externa carece de significado. Por lo tanto, las aseveraciones de la metafísica carecen de significado. Éste es el argumento principal de Carnap (1950).

La segunda premisa es que un enunciado sólo tiene sentido cuando se interpreta como interno a un marco lingüístico. Como enunciado externo no tiene significado lingüístico; sin embargo, Carnap añade la siguiente aclaración: la única manera en la que las preguntas se pueden interpretar como externas y a la vez como preguntas con sentido es si se toman como "una pregunta práctica, una cuestión de decisión práctica que concierne la estructura de nuestro lenguaje." (Carnap, 1950: 207). En esta interpretación particular "los números existen", no es una aseveración acerca de la existencia de ciertas entidades, sino la expresión de la decisión práctica de adoptar un cierto lenguaje. "Los números existen" significaría, en este sentido, lo mismo que "Acepto un marco lingüístico donde cuantificamos sobre variables que toman como valores elementos de un dominio que contienen números". Esta decisión se debe hacer teniendo en cuenta criterios prácticos como la eficiencia, la productividad y la simplicidad de un marco lingüístico (Carnap, 1950: 214). Se trataría, por lo tanto, de un sentido metalingüístico del enunciado inicial. Ésta es la única manera en la que el enunciado "los números existen" se puede interpretar como externo, pero con sentido. Pero en esta interpretación no se trata de un enunciado teó- 
rico, y no es, estrictamente hablando, una aseveración que se puede evaluar como verdadera o falsa (Carnap, 1950: 214) . Al contrario, no es más que la expresión de una preferencia, y de una decisión práctica. Por otro lado, cualquier pregunta teórica, cuya respuesta es una aseveración, se puede hacer sólo después de la adopción de un marco lingüístico, y dentro de ese marco. El error que comete el metafísico es querer invertir este orden: el metafísico toma cuestiones ontológicas como previas e independientes a la introducción de un marco lingüístico. Considera que un marco teórico "es legítimo sólo si puede justificarse por una visión ontológica que suministra una respuesta afirmativa a la cuestión de la realidad" (Carnap, 1950: 214). Es decir, según el metafísico, sólo podemos adoptar el marco teórico de las matemáticas si previamente podemos establecer la existencia real de los números.

Es interesante observar que Carnap da aquí un paso hacia el pragmatismo. A la vez que insiste en que las aseveraciones metafísicas no tienen un contenido teórico (aunque no explica qué quiere decir exactamente con eso), admite que se pueden re-interpretar como aseveraciones prácticas. Escribe Carnap:

A menos que, y no antes de que, [los metafísicos] suministren una interpretación cognitiva clara, estamos justificados en nuestra sospecha de que su pregunta es una pseudopregunta, es decir, una pregunta disfrazada en forma de una cuestión teórica,

6 Tal como señala Huw Price (1997: 11), preguntar ¿existen los números?, como pregunta teórica, "es un poco como intentar preguntar si las reglas de un juego son ciertas. Por ejemplo, alguien podría decir: 'Los jugadores de ajedrez dan por supuesto que el tablero de ajedrez tiene sesenta y cuatro casillas, ¿pero es éste realmente el caso?'”. Es hacer una pregunta acerca de las reglas del juego como si se tratara de un asunto de hecho. 
mientras que, de hecho, es no-teórica; en el caso presente es el problema práctico de incorporar o no al lenguaje las nuevas formas lingüísticas que constituyen el marco de los números. (Carnap, 1950: 209).

Por lo cual Carnap no niega que los enunciados de la metafísica tengan contenido, sólo niega que tengan el contenido que el metafísico cree darles.

Quine, por su lado, afirma que va todavía más lejos en este sentido al sostener un pragmatismo de mayor alcance. Con base en su rechazo de la distinción entre lo analítico y lo sintético, Quine rechaza distinciones como la de Carnap entre cuestiones pragmáticas acerca de la adecuación de un cierto lenguaje y cuestiones teóricas, acerca de los hechos que una teoría pretende describir. Lo expresa claramente en el siguiente fragmento de "Dos dogmas del empirismo" (1951a): "Carnap ha sostenido que ésta [la pregunta de si aceptar las clases como entidades] es una cuestión no de realidad sino de la elección de una forma de lenguaje conveniente, un esquema o marco conceptual conveniente para la ciencia. Con esto estoy de acuerdo, pero sólo con la condición de que lo mismo se conceda con respecto a las hipótesis científicas en general"' (Quine, 1951a: 45-46) 7 .

Apoyándose en la distinción entre preguntas externas e internas, Carnap rechaza el criterio de compromiso ontoló-

$7 \quad$ A continuación, Quine escribe: “Carnap ha reconocido que es capaz de mantener un doble estándar para cuestiones ontológicas e hipótesis científicas sólo asumiendo una distinción absoluta entre lo analítico y lo sintético; y no tengo que decir una vez más que se trata de una distinción que rechazo... Carnap, Lewis, y otros toman una posición pragmática sobre la cuestión de la elección entre las formas lingüísticas, los marcos científicos; pero su pragmatismo acaba en la frontera imaginaria entre lo analítico y lo sintético. Al repudiar esa frontera adopto un pragmatismo más exhaustivo" (1951a: 45-46). La misma observación la hace en Word and Object (1960: 271). 
gico que propone Quine. El compromiso ontológico (en el sentido de la metafísica tradicional) de una teoría con ciertas entidades no resulta inmediatamente de la cuantificación sobre variables que reciben su valor en un dominio de entidades de ese tipo. Pensar eso sería, según Carnap, confundir el sentido interno de un enunciado con el sentido externo (el que le quiere dar el metafísico). "Los números existen", como enunciado interno a las matemáticas, es verdadero, pero no implica platonismo acerca de la existencia de los números. Escribe Carnap: "No debe considerarse que la aceptación de un marco lingüístico implique una doctrina metafísica sobre la realidad de las entidades en cuestión. Me parece que debido al descuido de esta importante distinción algunos nominalistas contemporáneos etiquetan la admisión de variables de tipo abstracto como Platonismo. Ésta es, por decir lo menos, una terminología muy engañosa" (Carnap, 1950: 214-215). En la nota al pie número 5 a este fragmento, Carnap menciona a Quine (1948) como ejemplo de alguien que comete este error.

Antes de pasar a una discusión del argumento de Carnap en el siguiente apartado, mencionaré brevemente los siguientes pasos del debate entre Carnap y Quine. El artículo de Quine, “On Carnap's Views on Ontology" (1951b), es una influyente crítica al argumento de Carnap (1950). Quine desarrolla allí dos objeciones. Una de ellas es que la distinción de Carnap entre enunciados internos y externos no se sostiene, ya que presupone la distinción entre enunciados analíticos y sintéticos. Y esta última distinción, aunque tiene cierta fuerza intuitiva, es ininteligible, según el argumento de Quine de "Dos dogmas...". Es relevante recordar en este contexto que Quine rechaza toda una familia de conceptos, incluyendo el de analiticidad, sinonimia, significado 
e intensión. Estas nociones forman un "círculo" de conceptos, de modo que cualquiera de ellas puede ser definida a partir de las otras, pero ninguna de ellas tiene condiciones de aplicación claras.

Carnap intuye esta objeción de Quine y la menciona ya en Carnap (1950: 215, fn. 5); sin embrago, su réplica llegará sólo en "Meaning and Synonymy in Natural Language" (1955). Sostiene allí que la noción de intensión de una expresión es filosóficamente tan respetable como la de extensión, pues puede recibir criterios de aplicación igual de claros. La intención de una expresión se puede caracterizar considerando la evidencia empírica que se obtiene acerca de la respuesta de sujetos lingüísticamente competentes a preguntas acerca del valor de verdad de ciertos enunciados, considerándolos en diferentes circunstancias de evaluación. Podemos distinguir así entre las intensiones de predicados que tienen la misma extensión, como "unicornio" y "duende". Éstos, aunque tengan la misma extensión en el mundo actual, el conjunto vacío, tienen extensiones diferentes respecto a otras circunstancias de evaluación (véase Carnap, 1955: 236-9); por lo tanto, sus intensiones, definidas como funciones de circunstancias de evaluación a extensiones, son diferentes. Esta diferencia se ve reflejada en la conducta lingüística de los hablantes competentes puestos en contextos, en los cuales las diferencias se vuelven relevantes para la evaluación del enunciado. Así es posible caracterizar la noción de intensión de manera rigurosa. Posteriormente, podemos caracterizar las demás nociones del 'círculo' de manera rigurosa, pues se pueden definir a partir de la noción de intensión. En los escritos posteriores, tal como Word and Object (1960), Quine no rechazará el condicional según el cual si la extensión es una noción filosóficamente respetable, también lo es la in- 
tención. Pero usará la conversa: como la intensión no es una noción aceptable, tampoco lo es la extensión. Y esto, tal como señala Soames (2008: 17), le llevó “por el camino desastroso que conduce a sus tesis esencialmente eliminativistas -de la indeterminación de la traducción y la inescrutabilidad de la referencia".

Quine (1951b) desarrolla una segunda objeción al argumento de Carnap (1950), que resumiré brevemente. Señalé antes que Carnap compara preguntas como: “¿existe un número mayor que 5 y menor que 7?", con preguntas como, “¿existen los números?”. Estas últimas, dice Carnap, pueden entenderse bien como cuestiones internas, y en ese caso tienen significado, o como cuestiones externas, en cuyo caso se trata de un enunciado que sólo aparentemente expresa una proposición, pero que es en realidad sin sentido. Según Quine (1951b: 130):

[...] la dicotomía de Carnap entre distintas cuestiones de existencia es una dicotomía entre las preguntas de la forma "¿Existen tales-ytales cosas?", donde los tales-y-tales se usan para agotar el dominio de un estilo particular de variables ligadas, y las preguntas de la forma “ ¿Existen tales-y-tales cosas?" donde los tales-y-tales no se usan para agotar el dominio de un estilo particular de variables ligadas. Llamaré a las primeras preguntas categoriales [category questions], y a las últimas preguntas de subclase [subclass questions].

Además, Quine considera que la posibilidad de tener tanto una interpretación interna como una interpretación externa sólo existe para preguntas categoriales. Una pregunta de subclase no puede interpretarse en sentido externo. Efectivamente todos los ejemplos de preguntas externas que da 
Carnap son ejemplos de preguntas categoriales; sin embargo, observa Quine, la diferencia entre preguntas categoriales y preguntas de subclase depende de "irrelevantes cambios tipográficos" (Quine, 1951b: 133), detalles irrelevantes acerca de la configuración del lenguaje que usamos. Consideremos un lenguaje de primer orden, en cuya semántica el dominio de individuos contiene tanto números como objetos físicos. En este caso podemos elegir tener en nuestro lenguaje un único tipo de variables, que recibe como valor semántico cualquier elemento del dominio, o introducir un tipo de variables que recibe como valor números, y otro tipo de variables que recibe como valor objetos físicos. En el primer caso, “¿Existen los números?” es una pregunta categorial, mientras que en el segundo resulta ser una pregunta de subclase. Si esta lectura de Carnap es correcta, entonces sólo en el primer caso la pregunta se puede interpretar como externa y, por lo tanto, sin sentido. Si la pregunta del metafísico es o no externa depende de si es o no categorial (cuando se interpreta como interna a las matemáticas), y esto a su vez depende de si escogemos un estilo único de variables o no. Pero esta elección no tiene ninguna importancia: se puede pasar de un estilo uniforme de variables a una multiplicidad de estilos sin ninguna pérdida de poder expresivo.

Esta objeción de Quine tampoco logra su propósito. Suscribo a la observación de Haack (1976: 468), Bird (1995: 4849), y Thomasson (en preparación) según la cual Quine se equivoca al suponer que la distinción interno-externo depende de la distinción subclase-categorial. ¿Por qué pensar que sólo las preguntas categoriales se puedan interpretar como externas? Carnap nunca dice tal cosa en el artículo citado. Es verdad, sin embargo, que sus ejemplos de preguntas externas son preguntas de máxima generalidad, es decir, pregun- 
tas categoriales. Pero eso se debe a que Carnap enfoca sobre todo los enunciados externos tal como se encuentran en la metafísica, y esos enunciados son de carácter máximamente general, es decir, categoriales. Dejando de lado el enfoque de Carnap en la metafísica, no hay razón para pensar que lo que resulta ser un enunciado o una pregunta de subclase cuando se interpreta internamente a un lenguaje, no pudiera interpretarse externamente a ese lenguaje, es decir, sin apelar a las reglas sintácticas y semánticas de ese lenguaje.

Dejo aquí la parte expositiva y pasaré a desarrollar la objeción que considero que muestra que el argumento de Carnap no sostiene su conclusión.

¿Por qué falla el argumento de Carnap?

De entrada, se imponen unas observaciones terminológicas. Tal como hemos visto, Carnap distingue dos sentidos en los que uno puede entender una pregunta particular (o un enunciado); sin embargo, es mejor no hablar en este contexto de dos sentidos del enunciado en cuestión, ya que sería una petición de principio en contra de la tesis de Carnap de que las cuestiones externas no tienen sentido. Además, por la misma razón, no deberíamos hablar de preguntas (y enunciados) ambiguas (aunque Carnap lo hace, 1950: 213). Por lo general, se dice de las oraciones que son ambiguas si tienen dos significados cuando se interpretan de acuerdo con las reglas semánticas de un mismo idioma. La situación aquí es diferente, ya que una forma de palabras recibe dos interpretaciones de acuerdo a las reglas sintácticas y semánticas de dos idiomas diferentes. Usaré aquí el término de interpretación, en el sentido en que decir que una oración es interpretable no es decir que la interpretación le asigna un contenido semántico, sino simple- 
mente que hay posibilidad de dar sentido al acto de proferir la oración. Carnap no niega que cuando se toman como externas a un marco lingüístico las oraciones son interpretables en este sentido. Sólo niega que expresen una proposición que se pueda evaluar como verdadera o falsa, es decir, que tengan condiciones de verdad, o contenido semántico. De hecho, Carnap afirma explícitamente que las oraciones cuando se consideran como externas a un marco lingüístico son interpretables, pues se pueden interpretar como expresando la decisión del hablante de adoptar un cierto marco lingüístico. Con lo cual me parece acertado hablar de distintas interpretaciones -algunas de las cuales, según Carnap, no asignan al enunciado un contenido semántico, sino que indican que literalmente es sin sentido.

Como punto terminológico adicional, probablemente no deberíamos decir tampoco que lo que se interpreta es un enunciado o una pregunta. Esta manera de hablar presupone que ya hemos interpretado sintácticamente la cadena de huellas en el papel, o de sonidos oídos, y la hemos identificado como siendo una ocurrencia de una expresión compleja en un determinado idioma; sin embargo, las reglas sintácticas de dos lenguajes no tienen por qué coincidir. Cuando la interpretación es interna a un cierto marco lingüístico se lleva a cabo apelando tanto a las reglas semántica como a las reglas sintácticas de ese lenguaje. Lo que interpretamos, estrictamente hablando, son huellas sobre papel o sonidos, no enunciados o preguntas; sin embargo, a falta de un término mejor, y por coherencia con la terminología que usa Carnap, hablaré aquí de interpretar preguntas y enunciados, teniendo en cuenta la presente advertencia.

Argumento a continuación que la estrategia de Carnap para generar sinsentidos no funciona. Según Carnap, para ob- 
tener la interpretación externa de un enunciado tal como "Los números existen", es esencial evitar en la interpretación las reglas semántica y sintáctica del respectivo marco lingüístico, en este caso, el de las matemáticas. Aun suponiendo que, contrario a lo que dice Quine, sí podemos distinguir claramente los marcos lingüísticos, y suponiendo que la interpretación que se le da en la metafísica a la pregunta “Existen los números?” es externa a las matemáticas, todavía no alcanzamos la conclusión esperada. Con esto todavía no tenemos la conclusión de Carnap de que los enunciados de la metafísica son sinsentidos, pues un enunciado no es un sinsentido por el mero hecho de ser externo a un marco lingüístico. Sólo una interpretación que es externa a todo marco lingüístico es una interpretación que no apela a ninguna regla sintáctica y semántica. Y sólo entonces se puede decir que es una interpretación que no puede proporcionar contenido semántico alguno, pues sin un marco lingüístico no hay interpretación semántica posible. El metafísico interpreta sus enunciados como externos al marco de las matemáticas, pero eso no quiere decir que los interpreta como externos a cualquier otro marco. La interpretación puede ser externa respecto a un marco lingüístico e interna respecto a otro. Este caso se da, por ejemplo, con una pregunta sencilla tal como “¿Cuál es la constitución interna de un tomate?". Se puede interpretar esta pregunta como interna al marco lingüístico de la biología, pero también como externa al marco de la biología e interna al marco de la química. En los dos casos, la interpretación le asigna a la pregunta un contenido semántico. De manera similar, se puede argumentar, en defensa de la metafísica tradicional, que la interpretación en este caso es externa a las matemáticas, pero no externa a cualquier marco lingüístico; al fin y al cabo, es una pregunta interna al marco de la metafísica. 
Así que algo más se necesita para llegar a la conclusión que Carnap desea defender. Se pueden identificar en el texto de Carnap indicios de que el autor piensa que la opción de considerar que la metafísica tiene su propio marco lingüístico no es viable. La idea sería que la metafísica tiene un estatus especial entre los discursos, en cuanto que la interpretación de sus oraciones es externa a todo marco lingüístico. No hay tal cosa como un marco lingüístico de la metafísica. La razón que sugiere Carnap es la siguiente: está en la naturaleza de la metafísica intentar responder a las preguntas más generales acerca de la naturaleza de las entidades que teorías científicas particulares postulan (los números de las matemáticas, las cuerdas de la teoría de cuerdas, las especies de la biología, etcétera). La metafísica considera los conceptos que las teorías científicas introducen y pregunta si hay algo en la realidad que estos conceptos nombran, para luego indagar acerca de cuál es la naturaleza de esas entidades, si se trata de bloques fundamentales de construcción de la realidad, etcétera. En las ciencias particulares los conceptos nuevos se introducen mediante postulados (o hipótesis), y se mantienen mientras resultan útiles para la investigación. Pero el metafísico quiere saber si los números, las cuerdas y las especies son reales en un sentido extra-teórico; no si las matemáticas cuantifican sobre números y la biología sobre especies, sino si realmente existen tales cosas. Al hacer esto, el metafísico da un paso fuera de cualquier teoría particular. Es decir, la interpretación metafísica de la pregunta es externa a todos los marcos teóricos.

Pero esta línea de argumentación todavía no es suficiente para respaldar la conclusión de que no hay tal cosa como un marco lingüístico de la metafísica. Se puede sostener que las afirmaciones metafísicas son en efecto externas a los marcos de cualquier teoría científica particular, en el sentido de que 
las oraciones metafísicas no deben ser interpretadas y evaluadas por apelación al lenguaje y los métodos de evaluación de ninguna ciencia particular. Pero esto no quiere decir que no hay un posible marco lingüístico en el que afirmaciones metafísicas pueden realizarse e interpretarse (ni siquiera que no hay un marco lingüístico actual). A primera vista, el discurso metafísico es significativo, ya que parece que lo entendemos. Desde un punto de vista fenomenológico en los debates metafísicos, el lenguaje se emplea con significado. Si se trata meramente de una ilusión de pensamiento, se necesita un argumento para mostrar que eso es así, y Carnap nunca lo proporciona. Todo lo que encontramos en el texto de Carnap son declaraciones como la siguiente: “Desafortunadamente, estos filósofos no han dado hasta ahora una formulación de su pregunta en términos del lenguaje científico común. Por lo tanto, nuestro juicio debe ser que no han conseguido dar a la cuestión externa y a las respuestas posibles ningún contenido cognitivo" (Carnap, 1950: 209).

La cuestión esencial es la cuestión sobre por qué los enunciados de la metafísica, al ser externos a las ciencias, son sinsentidos. Y a esta pregunta Carnap no contesta. Si hay algo profundamente equivocado en la investigación que emprende el metafísico, Carnap no nos dice qué es. El que sea un discurso externo al de las ciencias no es en sí mismo un criterio para considerar la metafísica un conjunto de enunciados sin sentido. En conclusión, el argumento de Carnap no consigue mostrar que las afirmaciones de la metafísica no tienen sentido. En última instancia, esto requeriría que demuestre que el lenguaje de la metafísica carece de una semántica adecuada. Pero no da ningún argumento para apoyar esta afirmación. El principio verificacionista que Carnap defendió en la década de 1930 era lo que sujetaba el 
peso de la demostración de que el lenguaje metafísico carece de sentido. En la década de 1950 no encontramos ningún criterio análogo de significado lingüístico (o contenido cognitivo) que juegue el mismo papel en la demostración. Con lo cual las pretensiones de mostrar conclusiones devastadoras respecto a la metafísica se quedan sin el respaldo necesario.

Consideraciones finales: ¿por dónde seguir buscando?

Intentaré en esta última sección evaluar el argumento de Carnap desde una perspectiva actual, en particular desde un punto de vista externista acerca de cómo se establece y mantiene el significado lingüístico. Asumo aquí, sin argumento, que el punto de vista externista es correcto, aunque no asumiré una versión detallada del externismo, sino solamente ciertas tesis que forman parte del núcleo de la teoría. Espero que esto se vea claramente a continuación.

El trasfondo teórico del que parte Carnap es el del verificacionismo, una teoría epistémica del significado, según la cual el significado literal de un enunciado (o de una proferencia de un enunciado, si tenemos en cuenta la dependencia contextual del significado literal) se identifica con las condiciones de contrastación empírica de ese enunciado. Según el externismo, el significado literal no es introspectivamente accesible al hablante, con lo cual, captar la proposición literalmente expresada no puede identificarse con la captación de las condiciones de verificación del enunciado. Pues los empiristas lógicos, al sostener que la proposición literal se caracteriza como condiciones de contrastación empírica pensaban sin duda que el hablante comprende y conoce cuáles son estas 
condiciones $^{8}$ (aunque el requisito fuera que el enunciado sea en principio contrastable, y no de hecho contrastable). Por lo tanto, la perspectiva que encontramos detrás del argumento de Carnap no es una perspectiva externista, sino internista.

De todas formas, habría que decir que el verificacionismo no es (o no parece ser) una premisa en el argumento de Carnap, y tampoco lo es el internismo. Pero si analizamos el argumento desde un punto de vista externista, es posible encontrar vías de desarrollo que no resultaban accesibles desde el internismo de Carnap; sin embargo, las perspectivas no resultarán muy prometedoras. Argumentaré que hay razones para pensar que los enunciados que Carnap trata como sinsentidos no lo son, aunque se dé el caso de que los términos que el metafísico usa sean externos a la metafísica. Pero antes, se imponen algunas observaciones acerca del externismo.

Las consideraciones externistas son metateóricas (o fundacionales, y no descriptivas). Esto quiere decir que no conforman una hipótesis acerca del significado de ciertas expresiones, sino una hipótesis acerca de los hechos en virtud de los cuales ciertas expresiones tienen el significado lingüístico que tienen. Por esa razón, estas consideraciones externistas son compatibles con varias teorías acerca de la semántica de las expresiones en cuestión.

La teoría externista acerca de cómo se establece y se mantiene el referente de un nombre propio, propuesta que se amplía al caso de los términos de género natural. Las consideraciones externistas imponen tres condiciones para que el uso de un nombre propio tenga un referente: 1) un bautismo inicial, mediante

8 Por ejemplo, Moritz Schlick escribe: “el conocimiento de cómo una proposición se verifica no es algo por encima de su comprensión verbal y lógica, sino que es idéntica con ella" (Schlick, 1936: 344). 
el cual el nombre se introduce en el lenguaje; 2) una cadena de usos de ese nombre, que preservan la referencia; 3 ) finalmente, por parte del hablante que adquiere la práctica lingüística de usar ese nombre, tiene que haber la intención de usarlo con la misma referencia que tiene el nombre cuando lo usan los demás hablantes. Me centraré sobre todo en esta última condición. Distintos autores la formulan de manera distinta, pero hay acuerdo respecto a la idea fundamental:

- Saul Kripke: “Cuando el nombre 'pasa de eslabón en eslabón', el receptor del nombre debe, creo, cuando lo aprende, intentar usarlo con la misma referencia que la persona de la cual lo ha oído" (Kripke, 1980: 96).

- Mark Sainsbury: "La decisión de un usuario nuevo de usar el nombre 'en el modo en el que se usa' por los usuarios existentes involucra una intención que puede expresarse así: Para todo $x$, si los usuarios de NN que yo encuentro refieren a $\mathrm{x}$, entonces usaré el nombre NN para referirme a x" (2005: 113).

- Scott Soames: “A fin de que uno sea un usuario de un nombre $n$ de un objeto $o$, se requieren dos cosas: (i) Uno debe haber adquirido una intención referencial que determina a $o$ como referente de $n$. Dos maneras en las que esto se puede lograr son escoger $n$ de otros que lo hayan usado como un nombre para $o$, e intentar usar $n$ para referirse a lo mismo que ellos; o independientemente estar en contacto con $o$ e introducir $n$ como un nombre de $o$. (ii) Uno debe entender que proferir de manera asertiva 'n es $\mathrm{F}^{\prime}$ es decir, del referente, o, de n que 'es F'" (2002: 70).

Estas condiciones de competencia para el uso de nombres propios, si se cumplen, ponen el hablante en situación de adquirir el nombre y añadirlo a su vocabulario. En los usos posteriores de este nombre, siempre cuando el hablante tiene las intencio- 
nes debidas, lo usa de manera competente y consigue referirse al objeto nombrado. Autores como los mencionados anteriormente rechazan explícitamente la postulación de condiciones de competencia que requieran la existencia de un conocimiento descriptivo del objeto nombrado por parte del hablante. Sainsbury, p. ej., argumenta que cuando uso un nombre como "Peter" con las intenciones debidas consigo referirme a lo que el nombre nombra, aunque esté equivocado acerca de la naturaleza del referente, y piense que Peter es una persona cuando en realidad es un caballo de carreras. Aun así, la referencia de "Peter" es Peter: "Un signo de que la iniciación ocurrió, aunque hubiera mucho que no haya entendido sobre la situación, es que podemos usar alegremente (y no sólo mencionar) el nombre 'Peter' en decir en qué consiste este fallo de comprensión: No he entendido que Peter era un caballo" (Sainsbury, 2005: 116).

Kripke (1980) y Putnam (1973) extienden esta concepción acerca de los hechos que fundamentan el significado de los nombres propios a los términos para géneros naturales. De esta manera, el significado de términos como "agua", "oro", los nombres de las especies de la biología, etcétera, tienen un significado en una comunidad lingüística sin que sea necesario que el hablante competente tenga una descripción que identifique de manera única el referente del término; sin embargo, observa Putnam, "Difícilmente podríamos usar palabras como «olmo» $\mathrm{o}$ «aluminio» si nadie tuviese una manera de reconocer olmos y aluminio; pero no todo aquél para el cual es importante la distinción tiene que ser capaz de hacerla [...]" (Putnam, 1973: 704). En particular, según La Hipótesis de la Universalidad de la División del Trabajo Lingüístico que propone Putnam, es suficiente que haya ciertos hablantes dentro de la comunidad que tengan la capacidad de dis- 
tinguir los olmos de las hayas, el aluminio del molibdeno, el oro de la pirita del hierro, etcétera. Los hablantes no expertos defieren a la comunidad de expertos, es decir, usan el término con la intención de referirse a aquello a lo que se refieren los expertos cuando usan ese término. Esta intención es la correspondiente para el caso de lo términos de género natural a la intención mencionada en los fragmentos de Kripke, Sainsbury y Soames citados anteriormente para el caso de los nombres propios.

La relevancia de este último punto para el argumento de Carnap es la siguiente. Según Carnap, el enunciado "Los números existen", tal como lo considera el metafísico, es un sinsentido. Resulta de la discusión del argumento de Carnap en las secciones anteriores que la razón que proporciona Carnap para considerar que un enunciado es sin sentido es que es externo a los marcos lingüísticos de las teorías particulares (las matemáticas en el caso de "número", la biología en el caso de "especie", etcétera). Ahora bien, en el caso del enunciado "Los números existen", si aceptamos que el significado del cuantificador existencial no es cuestionable, y presuponiendo el principio de composicionalidad, la conclusión a la que llegamos es que el uso del término "número" por parte del metafísico es el que no tiene significado lingüístico. Por lo tanto, según Carnap, el uso del término "número" por parte del metafísico no tiene significado lingüístico precisamente porque es un término externo a las matemáticas. Pero, tal como he argumentado en las secciones anteriores, no hay razón alguna para pensar que si el uso de una expresión es externa a un marco lingüístico se trata de un uso que carece de significado. Esto parece ser correcto también para el caso de los términos: no hay razón (o, por lo menos, Carnap no nos proporciona razón alguna) para pensar que si el uso de un término es externo a un lenguaje, es un uso sin sentido. 
Esta conclusión se ve ahora reforzada por las consideraciones externistas introducidas más arriba. El que un término se use fuera del ámbito lingüístico de la comunidad de expertos no proporciona razón alguna para sostener que es un uso sin sentido. Por el contrario, si consideramos la hipótesis de Putnam acerca de la división del trabajo lingüístico, es suficiente que el hablante no experto use el término con la intención debida, en particular, con la intención de significar lo que los expertos significan con el término, para darle el significado debido. En nuestro caso, es suficiente que el metafísico tenga la intención de usar el término "número" con el significado que éste tiene en las matemáticas (es decir, para referirse a lo que los matemáticos se refieren con "número"), el término "especie" con el significado que tiene en la biología, y así en adelante. Si esto ocurre, el uso que hace de estos términos tiene el significado que éstos tienen en las respectivas disciplinas mencionadas. Aunque el uso por parte del metafísico de estos términos sea externo a las matemáticas, el metafísico no se encuentra en imposibilidad de formar las intenciones debidas, las que la teoría metasemántica externista requiere que tenga para usar el término con sentido. Con lo cual podemos concluir que el enunciado "Los números existen" es un enunciado significativo incluso cuando lo usa el metafísico.

En la sección anterior he argumentado que Carnap no proporciona razón alguna para pensar que el uso de preguntas como “¿Existen los números?” y de las posibles respuestas es carente de significado lingüístico. En esta última sección he argumentado que la teoría metasemántica externista proporciona una razón positiva para pensar que el uso de estas preguntas y respuestas en la metafísica no da lugar a sinsentidos. Por lo tanto, la posición de Carnap respecto a 
estas preguntas y enunciados es difícilmente sostenible; sin embargo, creo que cabe la posibilidad de una vía de desarrollo del argumento de Carnap. Se trataría de una estrategia de detectar el sinsentido inspirada tanto en el argumento de Carnap como en las consideraciones externistas mencionadas anteriormente. Pero esta estrategia no tendría efectos en cuanto a los enunciados existenciales tal como "Existen números", y a las preguntas correspondientes, sino sólo para enunciados con estructura más compleja. Dejaré el desarrollo de este argumento para otra ocasión.

\section{Bibliografía}

Almog, J. (1984). Semantical Anthropology. Midwest Studies in Philosophy, 9(1), 478-489.

Armstrong, D. (1989). Universals: An Opinionated Introduction. Westview Press.

Barnes, E. (2009). Review of Metametaphysics: New Essays on the Foundations of Ontology. En David Chalmers, David Manley y Ryan Wasserman (eds.). Notre Dame Philosophical Reviews.

Bird, G.H. (1995). Carnap and Quine: Internal and external questions. Erkenntnis, 42(1): 41-64.

Carnap, R. (1956). Meaning and Necessity (2a ed. with supplementary essays). Chicago: University of Chicago Press.

Carnap, R. (1955). Meaning and Synonymy in Natural Language. Philosophical Studies, 6, 33-47; repr. in Carnap (1956), 233-247.

Carnap, R. (1950). Empiricism, Semantics and Ontology. Revue International de Philosophie. 4, 20-40; repr. in Carnap 1956, 205-221. 
Carnap, R. (1935). Philosophy and Logical Syntax. Londres: Kegan Paul.

Carnap, R. (1932). Überwindung der Metaphysik durch logische Analyse der Sprache. Erkenntnis. 2: 219-241; trad. A. Pap: The Elimination of Metaphysics through Logical Analysis of Language. En Ayer (ed.). (1959). Logical Positivism. New York: Free Press, 60-81.

Evans, G. (1982). The Varieties of Reference. Oxford: Oxford University Press.

Haack, S. (1976). Some preliminaries to ontology. Journal of Philosophical Logic, 5(4), 457-474.

Hempel, C. (1950). Problems and Changes in the Empiricist Criterion of Meaning. Revue Internationale de Philosophie, 41(11), 41-63.

Kripke, S. (1980). Naming and Necessity. Cambridge: Harvard University Press.

Price, H. (1997). Carnap, Quine and the Fate of Metaphysics. The Electronic Journal of Analytic Philosophy, Issue 5 (Spring).

Putnam, H. (1973). Meaning and Reference. The Journal of Philosophy, 70(19), 699-711.

Quine, W.O. (1947). On Universals. J. Symbolic Logic. Volume 12 , Issue $3,74-84$.

Quine, W.O. (1960). Word E Object. Cambridge, Mass: MIT Press.

Quine, W.O. (1953a). From a Logical Point of View. Cambridge, Mass.: Harvard University Press.

Quine. W.O. (1953b). Logic and The Reification of Universals. En From a Logical Point of View, 102-130.

Quine, W.O. (1951a). Two Dogmas of Empiricism. Philosophical Review. 60, 20-43; reprinted in From a Logical Point of View, 20-46. 
Quine, W.O. (1951b). On Carnap's Views on Ontology. Philosophical Studies 2.

Quine, W.O. (1948). On What There Is. Review of Metaphysics. Reimpreso en From a Logical Point of View, 1-20.

Sainsbury, M. (2005). Reference Without Referents. Oxford: Clarendon Press.

Schlick, M. (1936). Meaning and Verification. The Philosophical Review, 45(4), 339-369.

Sellars, W. (1975). Autobiographical Reflections. En Héctor Neri Castañeda (ed.). Action, Knowledge, and Reality: Critical Studies in Honor of Wilfrid Sellars. Indianapolis: The Bobbs-Merrill Company, Inc.

Soames, S. (2002). Beyond Rigidity: The Unfinished Semantic Agenda of 'Naming and Necessity. Oxford: Oxford University Press. Thomasson A.L. (en preparación). Carnap and the Prospects for Easy Ontology. En Blatti, Stephan y LaPointe, Sandra (eds.). Ontology after Carnap. Oxford: Oxford University Press. 


\section{Resumen}

En el artículo "Empirismo, Semántica y Ontología" (1950), Rudolf Carnap propone un argumento a favor de la conclusión de que ciertos enunciados, algunos pertenecientes a la metafísica, carecen de significado lingüístico. El artículo se discute en general como parte de un debate más amplio entre Carnap y Quine en la década de 1950. En este trabajo me propongo hacer tres cosas: en primer lugar, presentar brevemente el debate, con el fin de aclarar los propósitos dialécticos de Carnap en el artículo mencionado; en segundo lugar, argumentar que la estrategia de Carnap de detectar el sinsentido, falla; y por último, analizar el argumento de Carnap desde una perspectiva metasemántica externista.

Palabras clave: Carnap, sinsentido, preguntas, externas, internas.

\section{Abstract}

In his seminal article "Empiricism, Semantics and Ontology" (1950) Rudolf Carnap proposes an argument to support the conclusion that certain sentences, among which some that belong to metaphysics, lack linguistic meaning. Carnap's article is generally discussed as part of a wider debate between Carnap and Quine during the 50's. In this paper my aim is threefold: first, to briefly review the Carnap-Quine debate, with the purpose of clarifying Carnap's dialectical purposes in the article mentioned; second, I argue that Carnap's strategy for detecting meaninglessness fails; and finally, I suggest a possible way in which Carnap's argument could be recast in externalist meta-semantic terms.

Keywords: Carnap, meaningless, external, internal, questions. 
